\title{
An Electron-Hole Transport Model for the Analysis of the Photorefractive Harmonic Gratings
}

\author{
P. Vaveliuk, B. Ruiz, O. Martinez Matos, G. A. Torchia, and N. Bolognini
}

\begin{abstract}
The steady-state exact solution for the higher harmonic gratings that synthesize the space-charge field is derived without restrictions within an electron-hole transport model which allows the behavior of these harmonic gratings to be determined rigorously in terms of the main photorefractive parameters. The model predicts the independence of the fundamental and harmonic amplitudes on the average excitation intensity. With respect to the modulation depth $m$, the dependence of each $\nu$-harmonic order is established as $m^{\nu}$ which is the result obtained in the single-level model. In terms of the grating spacing, three regions of quite different behavior are identified as the linear, transition, and nonlinear regions. The extent of each region in terms of $\Lambda$ strongly depends on the acceptor density relative to the donor density. If the acceptor density is much greater or smaller than the donor density, the linear region spreads out toward the lowest spacing, the nonlinear region extends toward the highest spacing, and the intermediate region is located in-between, as in the Kukhtarev model. But, for similar concentrations, the nonlinear region is shifted toward smaller spacing with respect to the linear region. On the other hand, the electron-hole competition can be deleterious for recording the grating, due to the charge compensation produced by the additional charge carrier that screens the internal space-charge field. Also, the relative importance of the higher harmonics is apparent for the smallest values of the external field as in the single-level model.
\end{abstract}

Index Terms-Electron-hole transport model, harmonic gratings, photorefractive materials.

\section{INTRODUCTION}

$\mathbf{T}$ HE STUDY of nonlinear effects in photorefractive materials is of great interest because of its potential applications in optical and optoelectronic devices. Experimentally, it is well established that the nonlinearity appears at large modulation depths of the excitation intensity [1] and the nonlinear phenomena are related to the loss of the sinusoidal profile of the refraction index grating, which was attributed to the formation of harmonic gratings in addition to the fundamental grating [2]. Each harmonic grating satisfies the Bragg condition for its fringe spacing and has been observed in several photorefractive materials, such as barium titanate $\left(\mathrm{BaTiO}_{3}\right)$ [3], [4], strontium barium niobate (SBN), barium strontium potassium sodium niobate (BSKNN) [3], bismuth oxide titanium (BTO) [5], and bismuth oxide silicon (BSO) [1], [6], [7]. From a theoretical point

Manuscript received January 9, 2001; revised April 23, 2001. The work of P. Vaveliuk and G. A. Torchia was supported by a CONICET fellowship. This work was supported by the Agencia Nacional de Promocion Cientifica Y Tecnologica (ANPCYT) under Grant PMT-PICT0041.

The authors are with Centro de Investigaciones Opticas (UNLP-CICBACONICET), cc 124 (1990) La Plata, Argentina, and also with the Universidad Nacional de La Plata, La Plata, Argentina.

Publisher Item Identifier S 0018-9197(01)05945-0. of view, each harmonic index grating is related to each harmonic term in the Fourier development of the space-charge field. The behavior of the higher harmonic field components was rigorously studied within the framework of the one-level model [8], [9]. However, for a diverse range of photorefractive materials, this early model fails to explain certain peculiar experimental results, as the sub-linear dependence of the photoconductivity on average excitation intensity [10] and double build-up and decay times in the diffraction efficiency [11]-[15] and photocurrent [12], [14], [16]. These results have been satisfactorily explained by means of the well-known shallow trap model, and the harmonic gratings were recently analyzed within this framework [18]. Unfortunately, this model does not cover all materials because there exist further experimental results that could not be explained by models that postulate a single-charge transport mechanism. Measurements of the gain coefficient for beam coupling, which is proportional to the space-charge field when there is no applied field, demonstrated that it changes its sign in terms of grating spacing and the excitation intensity in some photorefractive crystals [19]-[21]. This anomaly is necessarily attributed to the electron-hole competition process, and the sign of the space-charge field is related to the sign of the dominant charge transport carrier. Moreover, it was shown recently that the double exponential decay in the photocurrent, which was attributed to the shallow trap model, can also be explained by a model that postulates a bipolar transport mechanism [22]. Then, despite the fact that the electron-hole transport mechanism was extensively confirmed in certain photorefractive materials, the nonlinear regime, that must account for the appearance of higher harmonics, was not analyzed within this framework. A literature review indicates an isolated work that analyzes the relative behavior of the second harmonic grating by using an approximate resolution method of perturbative character [23].

The lack of awareness of the global behavior of the harmonic gratings within an electron-hole transport model suggested to us to analyze this approach over an arbitrary range of the main excitation parameters by solving in exact form the material equations for a model that postulates that electrons are generated from deep donor impurities and that holes are produced from deep acceptor species. Then, from the analysis, we predict the rigorous dependence of the harmonic amplitudes on the average intensity, modulation depth, grating spacing, and external field, as well as the precise space-charge field spatial distribution in the crystal and harmonic components needed for field convergence. Regions of linear and nonlinear behavior can also be clearly identified and the results can be compared with those obtained from unipolar transport models: specifically the Kukhtarev [9] and shallow trap models [18]. 


\section{Electron-Hole Transport Model}

The analysis is carried out by following an approach that postulates the existence of deep photorefractive centers that generate either an effective donor level in the forbidden gap, which is responsible for the generation and recombination of free electrons in the conduction band, or on the other hand, an effective acceptor level originated by other impurity centers, which is the responsible of the generation of holes in the valence band. This arrangement was observed in diverse photorefractive materials: BSO [24], $\mathrm{BaTiO}_{3}$ [19], [20], photorefractive semiconductors such as InP:Fe [23] and other materials [25], [26].

We assume that the steady-state gratings inside the crystal are formed by a stationary two-wave interference spatial pattern where the incident wave vectors form an isosceles triangle with the grating vector $\mathbf{K}$ of modulus $K=2 \pi / \Lambda$. The grating spacing is $\Lambda=\lambda / 2 \sin (\theta / 2)$, where $\lambda$ is the wavelength employed and $\theta$ is the angle between the incident wave vectors. The 1-D distribution intensity is

$$
I=I_{0}[1+m \cos (K x)]
$$

where $I_{0}=I_{1}+I_{2}$ and $I_{1}, I_{2}$ are the incident beam intensities, $m=2 \sqrt{I_{1} I_{2}} / I_{0}$ is the modulation depth, and $x$ is the spatial variable.

In the following, we will describe the electron-hole transport model concerned with this paper. It is essentially an enlarged version of the early Kukhtarev model [27]. The well-known transport mechanism processes, diffusion and drift, are taken into account for both charge carriers in this work. The absorption and two-wave coupling variation in the excitation beam propagation direction $z$ is disregarded so that the electron-hole model equations are reduced to $1-\mathrm{D}$ equations in the $x$-direction, perpendicular to the excitation direction $z$. The total current density $J$ is the sum of both density currents, $J_{e}$ for electrons and $J_{h}$ for holes, which take into account the drift in an electric field and diffusion due to a charge carrier concentration gradient

$$
\begin{aligned}
& J_{e}=e \mu_{e} n_{e} E-k_{B} \mu_{e} T \partial n_{e} / \partial x \\
& J_{h}=e \mu_{h} n_{h} E+k_{B} \mu_{h} T \partial n_{h} / \partial x
\end{aligned}
$$

where

$$
\begin{array}{ll}
n_{e} & \text { free-electron density in the conduction band; } \\
n_{h} & \text { free-hole density in the valence band; } \\
\mu_{e}, \mu_{h} & \text { electron and hole mobilities, respectively; } \\
e & \text { electronic charge; } \\
k_{B} & \text { Boltzmann's constant; } \\
T & \text { temperature; } \\
x & \text { spatial coordinate, perpendicular to the excitation di- } \\
& \text { rection. }
\end{array}
$$

$E=E_{0}+E_{\mathrm{sc}}$ is the total electric field, including the external and the raised photorefractive field.

The photorefractive material contains two noninteracting photoactive impurity species, donors of total number density $N_{D}$ and acceptors of total number density $N_{A}$. Electrons are ionized from donors of density $\left(N_{D}-N_{D}^{+}\right)$that move by drift and diffusion, and recombine into empty traps of number density $N_{D}^{+}$. Holes are photoexcited into the valence band from acceptors $\left(N_{A}-N_{A}^{-}\right)$, and recombine at traps denoted $N_{A}^{-}$. At thermal equilibrium, a certain fraction of donors and acceptors are ionized and there is a concentration of other nonactive ionized impurities of density $N_{D \text { eq }}^{+}$that compensates the ionized donors and $N_{A \text { eq }}^{-}$that compensates the acceptors. These impurity centers do not participate in the photorefractive process but ensure charge neutrality in the crystal. It can neglect both thermal reexcitation rates $\beta_{e}, \beta_{h}$, corresponding to electrons and holes, respectively, because the energy levels are deep. The population balance for donors and acceptors, Poisson's equation, and the electron and hole continuity equations can be written as [27]

$$
\begin{aligned}
\frac{\partial N_{D}^{+}}{\partial t} & =s_{D} I\left(N_{D}-N_{D}^{+}\right)-\gamma_{D} n_{e} N_{D}^{+} \\
\frac{\partial N_{A}^{-}}{\partial t} & =s_{A} I\left(N_{A}-N_{A}^{-}\right)-\gamma_{A} n_{h} N_{A}^{-} \\
\frac{\partial E}{\partial x} & =\frac{e}{\epsilon \epsilon_{0}}\left(n_{e}-n_{h}+N_{A}^{-}-N_{D}^{+}+N_{e q}\right) \\
\frac{\partial J_{e}}{\partial x} & =-e \frac{\partial}{\partial t}\left(n_{e}-N_{D}^{+}\right) \\
\frac{\partial J_{h}}{\partial x} & =e \frac{\partial}{\partial t}\left(N_{A}^{-}-n_{h}\right)
\end{aligned}
$$

where

$s_{D}, s_{A}$ cross sections for photoionization of deep donors and acceptors, respectively;

$\gamma_{D}, \gamma_{A}$ recombination constants of donors and acceptors, respectively;

$\epsilon \quad$ static dielectric constant;

$\epsilon_{0} \quad$ vacuum permittivity;

$N_{\text {eq }}=N_{A \text { eq }}^{-}-N_{D \text { eq }}^{+}$

Our aim is to obtain exact solutions for the higher harmonic amplitudes that synthesize the space-charge field in the steadystate regime from the equation set (1)-(8). Then, the rigorous behavior of the harmonic gratings can be analyzed within the electron-hole model framework.

\section{HaRmonic Gratings in Steady-State Regime}

The steady-state regime is obtained by setting $d / d t=0$ in the electron-hole material equations. In typical experiments, the spacing $\Lambda$ ranges between $0.2 \mu \mathrm{m}$ and $10 \mu \mathrm{m}$, which is much less than the crystal dimensions ( $\cong 10 \mathrm{~mm}$ ). Then, it is possible to disregard boundary effects and the variable $\Psi$, which represents any one of the magnitudes $E, J, N_{D}^{+}$and $n$, can be treated as being periodic with period $\Lambda$. Under these conditions, it is convenient to develop $\Psi$ in terms of a spatial Fourier series

$$
\Psi(x)=\psi_{0}+\sum_{\nu=1}^{\infty} \psi_{\nu} \cos \left(\nu K x+\varphi_{\nu}\right) .
$$

The problem is then reduced to find the grating amplitudes $\psi_{\nu}$, and phases $\varphi_{\nu}$, with regard to the excitation pattern $I(x)$. Math- 
ematically, it is more convenient to work in the complex plane. Then, we use the complex series [4]

$$
\Phi(x)=\phi_{0}+\sum_{\nu=1}^{\infty} \phi_{\nu} \exp (i \nu K x)
$$

to describe the real physical magnitudes. In addition, the variables $\Psi$ and $\Phi$ are related by $\Psi=\operatorname{Re}(\Phi)$ and the amplitudes and phases are related by $\psi_{\nu}=\left|\phi_{\nu}\right|$, and $\varphi_{\nu}=\arg \phi_{\nu}$. In particular, we are interested in the space-charge field Fourier expansion

$$
E_{\mathrm{sc}}(x)=\sum_{\nu=1}^{\infty} E_{\nu} \exp (i \nu K x)
$$

since, from the complex harmonic fields, $E_{\nu}=\left|E_{\nu}\right| e^{i \varphi_{\nu}}$, we can directly obtain the harmonic field component amplitudes and phases related to the photorefractive harmonic gratings. The diffracted intensity of each $\nu$-harmonic index grating is related to the amplitude of each $\nu$-harmonic component of the photorefractive space charge field [4], [8].

Before obtaining the $E_{\nu}$ gratings, we derive the equation for the stationary average free-carriers density. From (7)-(8)

$$
\begin{aligned}
& N_{D}^{+}-n_{e_{0}}=A_{1} \\
& N_{A}^{-}-n_{h_{0}}=A_{2}
\end{aligned}
$$

where $A_{1}$, and $A_{2}$ are constants. At thermal equilibrium, both free charge carrier densities are negligible because they must be excited from deep centers, i.e., $n_{e_{0}}=n_{h_{0}} \approx 0$, which indicates that $A_{1}=N_{D \text { eq }}^{+}$and $A_{2}=N_{A \text { eq. }}^{-}$. By combining (4)-(8), we obtain the equations of average density evolution for the free electrons and holes, which coincide with the equations obtained by Valley [27]

$$
\begin{aligned}
& \gamma_{D} n_{e_{0}}^{2}+\left(\gamma_{D} N_{D \text { eq }}^{+}+s_{D} I_{0}\right) n_{e_{0}}+s_{D} I_{0}\left(N_{D \text { eq }}^{+}-N_{D}\right)=0 \\
& \gamma_{A} n_{h_{0}}^{2}+\left(\gamma_{A} N_{A \text { eq }}^{-}+s_{A} I_{0}\right) n_{h_{0}}+s_{A} I_{0}\left(N_{A \text { eq }}^{-}-N_{A}\right)=0 .
\end{aligned}
$$

These equations reduce to the well-known single-level model equation [28], when acceptor species that generate holes become negligible with regard to the donors species that generates electrons, or vice versa [27]. The solutions are

$$
\begin{aligned}
n_{e_{0}}= & \left\{-\left(\gamma_{D} N_{D \text { eq }}^{+}+s_{D} I_{0}\right)\right. \\
& \left. \pm\left[\left(\gamma_{D} N_{D \text { eq }}^{+}-s_{D} I_{0}\right)^{2}+4 \gamma_{D} s_{D} I_{0} N_{D}\right]^{1 / 2}\right\} /\left(2 \gamma_{D}\right) \\
n_{h_{0}}= & \left\{-\left(\gamma_{A} N_{A \text { eq }}^{-}+s_{A} I_{0}\right)\right. \\
& \left. \pm\left[\left(\gamma_{A} N_{A \text { eq }}^{-}-s_{A} I_{0}\right)^{2}+4 \gamma_{A} s_{A} I_{0} N_{A}\right]^{1 / 2}\right\} /\left(2 \gamma_{A}\right)
\end{aligned}
$$

In the continuum regime with intensities between $10^{-3}-10$ $\mathrm{W} / \mathrm{cm}^{2}, n_{i 0} \ll N_{i \mathrm{eq}}$ and $n_{i_{0}}$ keeps a linear behavior in terms of $I_{0}$.
To find the exact solutions for the stationary harmonic gratings $E_{\nu}^{\text {st }}$, we reduce the original system of (2)-(8) to the simplest system given by the set of three equations as follows:

$$
\begin{aligned}
& \frac{d\left(n_{e}^{\mathrm{st}} E^{\mathrm{st}}\right)}{d x}=\frac{k_{B} T}{e} \frac{d^{2} n_{e}^{\mathrm{st}}}{d x^{2}}, \quad \frac{d\left(n_{h}^{\mathrm{st}} E^{\mathrm{st}}\right)}{d x}=-\frac{k_{B} T}{e} \frac{d^{2} n_{h}^{\mathrm{st}}}{d x^{2}} \\
& {\left[\gamma_{D} n_{e}+s_{D} I_{0}(1+m \exp (i K x))\right]} \\
& \quad \times\left[\gamma_{A} n_{h}+s_{A} I_{0}(1+m \exp (i K x))\right] \\
& \quad \times\left(n_{e}^{\mathrm{st}}-n_{h}^{\mathrm{st}}+N_{\mathrm{eq}}-\frac{\epsilon \epsilon_{0}}{e} \frac{d E^{\mathrm{st}}}{d x}\right) \\
& \quad=s_{D} I_{0} N_{D}(1+m \exp (i K x)) \\
& \quad \times\left[\gamma_{A} n_{h}+s_{A} I_{0}(1+m \exp (i K x))\right] \\
& \quad-s_{A} I_{0} N_{A}(1+m \exp (i K x)) \\
& \quad \times\left[\gamma_{D} n_{e}+s_{D} I_{0}(1+m \exp (i K x))\right] .
\end{aligned}
$$

Then, by replacing series (10) in the system (18)-(19) and after tedious calculations, equations in the different $\exp (i \nu K x)$ factors are generated $(\nu=1, \ldots, \infty)$. By equalizing coefficients of the same order in $\exp (i \nu K x)$, it is possible to obtain expressions for the complex harmonic field coefficients $E_{\nu}^{\text {st }}$ in terms of material and excitation parameters, the $\nu$-order carrier grating, $n_{\nu}$, and the lowest orders of the harmonic fields and carrier gratings $E_{j}, n_{j}$ with $j=\nu-1, \ldots, 1$. The expressions for the $\nu$-order carrier gratings $n_{\nu}^{\text {st }}$ are

$$
\begin{aligned}
& n_{e_{\nu}}^{\mathrm{st}}=-\frac{i}{\left(\nu E_{D}+i E_{0}\right)} \sum_{j=0}^{\nu-1} n_{j}^{\mathrm{st}} E_{\nu-j}^{\mathrm{st}}, \quad \nu \geq 1 \\
& n_{h_{\nu}}^{\mathrm{st}}=\frac{i}{\left(\nu E_{D}-i E_{0}\right)} \sum_{j=0}^{\nu-1} n_{j}^{\mathrm{st}} E_{\nu-j}^{\mathrm{st}}, \quad \nu \geq 1 .
\end{aligned}
$$

The fundamental, second, and arbitrary $\nu$-order field harmonic amplitudes $(\nu \geq 3)$ can be written as

$$
\begin{aligned}
E_{1}^{\mathrm{st}}= & \frac{i m\left(E_{D}+i E_{0}\right)}{M_{1}}\left[\left(1-r_{2}\right) E_{Q_{D}}-\left(1-r_{1}\right) E_{Q_{A}}\right] \\
E_{2}^{\mathrm{st}}= & \frac{\left(2 E_{D}+i E_{0}\right)}{M_{2}} \\
& \times\left\{i m^{2}\left[\left(1-r_{2}\right) r_{1} E_{Q_{D}}-\left(1-r_{1}\right) r_{2} E_{Q_{A}}\right]-m E_{1}^{\mathrm{st}}\right. \\
& \times\left(\left[\left(1-r_{1}\right) /\left(E_{D}-i E_{0}\right)+r_{1} /\left(E_{D}+i E_{0}\right)\right]\left(1-r_{2}\right)\right. \\
& \times E_{Q_{D}}+\left[\left(1-r_{2}\right) /\left(E_{D}+i E_{0}\right)+r_{2} /\left(E_{D}-i E_{0}\right)\right] \\
& \left.\times\left(1-r_{1}\right) E_{Q_{A}}\right)+i\left(E_{Q_{A}}-E_{Q_{D}}\right)\left(1-r_{1}\right)\left(1-r_{2}\right) \\
& \times\left(E_{1}^{\mathrm{st}}\right)^{2} /\left(E_{D}^{2}+E_{0}^{2}\right)-E_{1}^{\mathrm{st}} m\left(r_{1}+r_{2}\right)+i\left(E_{1}^{\mathrm{st}}\right)^{2} \\
& \times\left[\left(1-r_{2}\right) /\left(E_{D}+i E_{0}\right)-\left(1-r_{1}\right) /\left(E_{D}-i E_{0}\right)\right] \\
& +i\left(E_{1}^{\mathrm{st}}\right)^{2}\left[\left(1-r_{2}\right) E_{Q_{D}} /\left(E_{D}+i E_{0}\right)\right. \\
& \left.\left.-\left(1-r_{1}\right) E_{Q_{A}} /\left(E_{D}-i E_{0}\right)\right]\right\}
\end{aligned}
$$




$$
\begin{aligned}
\text { and } & \\
E_{\nu}^{\mathrm{st}}= & \frac{i\left(\nu E_{D}+i E_{0}\right)}{M_{\nu}}\left\{m E_{Q_{D}}\left(1-r_{2}\right)\right. \\
& \times\left[\left(1-r_{1}\right) R_{\nu-1}^{*}-r_{1} R_{\nu-1}\right]-m E_{Q_{A}}\left(1-r_{1}\right) \\
& \times\left[\left(1-r_{2}\right) R_{\nu-1}-r_{2} R_{\nu-1}^{*}\right]+\left(E_{Q_{A}}-E_{Q_{D}}\right) \\
& \times\left(1-r_{1}\right)\left(1-r_{2}\right) \sum_{j=1}^{\nu-1} R_{j} R_{\nu-j}^{*} \\
& +i \sum_{j=1}^{\nu-1} j E_{j}\left[\left(1-r_{1}\right) R_{\nu-j}^{*}+\left(1-r_{2}\right) R_{\nu-j}\right. \\
& \left.-\left(1-r_{1}\right)\left(1-r_{2}\right) \sum_{h=2}^{\nu-j-2} R_{h} R_{\nu-j-h}^{*}\right] \\
& +i \sum_{j=1}^{\nu-1} j E_{j}^{\mathrm{st}}\left[\left(1-r_{1}\right)\left(m r_{2}+\left(1-r_{2}\right) R_{1}\right) R_{\nu-j-1}^{*}\right. \\
& \left.+\left(1-r_{2}\right)\left(m r_{1}+\left(1-r_{1}\right) R_{1}^{*}\right) R_{\nu-j-1}\right] \\
& +i(\nu-2) E_{\nu-2}\left(m r_{2}+\left(1-r_{2}\right) R_{1}\right) \\
& \times\left(m r_{1}+\left(1-r_{1}\right) R_{1}^{*}\right)+i(\nu-1) E_{\nu-1} m\left(r_{1}+r_{2}\right) \\
& -\sum_{j=1}^{\nu-1} E_{j}\left[\left(1-r_{1}\right) E_{Q_{A}} R_{\nu-j}^{*}\left(\nu E_{D}+i E_{0}\right) /\right. \\
& \left.\left.\times\left(\nu E_{D}-i E_{0}\right)+\left(1-r_{2}\right) E_{Q_{D}} R_{\nu-j}\right]\right\} \\
& \left.E_{D}\right\}
\end{aligned}
$$

where $E_{D}=K k_{B} T / e$ is the diffusion field, $E_{Q_{I}}=$ $e N_{i \mathrm{eq}} / K \epsilon_{0}$ is the donor $(i=D)$ or acceptor $(i=A)$ saturation field, and

$$
\begin{aligned}
M_{\nu}= & \nu\left(\nu E_{D}+i E_{0}\right)+\left(1-r_{2}\right) E_{Q_{D}} \\
& +\left(1-r_{1}\right) E_{Q_{A}}\left(\nu E_{D}+i E_{0}\right) /\left(\nu E_{D}-i E_{0}\right) .
\end{aligned}
$$

The coefficients are $a_{0}=\beta_{S}+\gamma_{S} n_{0}, b_{0}=s_{D} I_{0}+n_{0} \gamma_{D}, a_{1}=$ $m \beta+n_{1}^{\mathrm{st}} \gamma_{S}, b_{1}=m s_{D} I_{0}+n_{1}^{\mathrm{st}} \gamma_{D}, a_{h}=\gamma_{S} n_{h}$ and $b_{h}=\gamma_{D} n_{h}^{\mathrm{st}}$, for $h \geq 2$. The coefficients $R_{h}$ are defined by recurrence as $R_{0}=1, R_{1}=-i E_{1} /\left(E_{D}+i E_{0}\right), R_{2}=$ $-i /\left(2 E_{D}+i E_{0}\right)\left(E_{2}+R_{1} E_{1}\right), \ldots, R_{h}=-i /\left(h E_{D}+\right.$ $\left.i E_{0}\right) \sum_{j=1}^{h-1} R_{j} E_{h-j}$.

\section{RESULTS AND DISCUSSION}

In this section, we discuss the results obtained from the analysis of the exact solutions of $E_{\nu}$ corresponding to the electron-hole transport model, given in (21)-(23). The simultaneous dependence of the photorefractive field components on the average excitation intensity $I_{0}$, the modulation depth $m$, and the grating spacing $\Lambda$, as well as the precise space-charge field spatial distribution within the material, with or without applied field, are analyzed for different concentrations of ionized and nonionized photorefractive species. The values of the experimental parameters are taken to be in the usual experimental range $10^{-3} \mathrm{~W} / \mathrm{cm}^{2} \leq I_{0} \leq 10 \mathrm{~W} / \mathrm{cm}^{2}, 0<m<1,0.1 \mu \mathrm{m}<$ $\Lambda \leq 10 \mu \mathrm{m}$ and $0 \leq E_{0} \leq 10 \mathrm{kV} / \mathrm{cm}$. This set of material parameters corresponds to that usually employed for BSO crystals [8], and has been used in our previous work concerning harmonic gratings within the one-level Kukhtarev model [9] and the shallow trap model [18].

\section{A. Harmonic Grating Behavior in Terms of the Main Photorefractive Parameters}

The behavior of harmonic amplitudes depends on the difference of the concentrations of impurity ionized and nonionized centers in thermal equilibrium that generate electrons and holes [24], [27], [29], [30]. The analysis is done by varying the concentration of impurities, that produces different rations of free electrons and holes. First, we analyze the dependence on the average excitation intensity. The harmonic gratings were predicted to be independent of $I_{0}$ for arbitrary parameter sets, as in the Kukhtarev model [9]. This behavior is related to the linear dependence of the photoconductivity on the intensity $I_{0}$.

In our previous papers related to the single and shallow trap models, it was clearly established that the higher gratings tend to appear more strongly for increasing values of both the modulation depth and the grating spacing. By following the same analysis within the electron-hole model, we depict in Fig. 1 the fundamental and the two higher harmonic amplitudes versus $\Lambda$ and $m$ simultaneously. Subfigures $\left(\mathrm{a}_{i}\right)$ and $\left(\mathrm{b}_{i}\right)$ represent the $E_{\nu}$-surfaces for different concentrations of donors and acceptors. The concentrations of ionized and nonionized acceptors responsible for hole generation are varied, and their numerical values are given in Fig. 1. Our simulations predict different behavior of the harmonic gratings for the different ranges of acceptor concentration. The grating amplitudes are shown to vary strongly in terms of thermal equilibrium ionized acceptors, $N_{A \text { eq }}^{-}$. Three typical behaviors appear depending on the $N_{A \text { aq }}^{-}$ values and also with regard to the thermal equilibrium ionized donors, $N_{D \text { eq }}^{+}$. When $N_{A \text { eq }}^{-} \gg N_{D \text { eq }}^{+}$, the photorefractive effect depends on the redistribution of holes in acceptor centers with the corresponding sign for the space-charge field. The Debye screening length $\Lambda_{D}$ is shifted toward lower $\Lambda$-values and the harmonic amplitudes of $E_{\mathrm{SC}}$ are enhanced around these values compared with the single-level model. The dynamical situation is equivalent to a model with a single photorefractive impurity center (acceptor) and single transport mechanism realized by holes with a high value of the thermal equilibrium ionized centers. It is observed that the $E_{1}$-surface changes very slightly with a strong variation of the total acceptor density $N_{A}$. For instance, the fundamental amplitude value, at Debye screening length $\Lambda_{D}$, varies only from $3.5 \mathrm{dV} / \mathrm{cm}$ for $N_{A}=5 \times 10^{17} \mathrm{~cm}^{-3}$, to $4.5 \mathrm{dV} / \mathrm{cm}$ for $N_{A}=5 \times 10^{19} \mathrm{~cm}^{-3}$. Identical behavior is observed for the higher harmonic gratings. On the other hand, when the condition $N_{A \text { eq }}^{-} \approx N_{D \text { eq }}^{+}$, is fulfilled [Fig. 1 $\left(\mathrm{a}_{i}\right)$ ], the distributions of electrons and holes are similar in the same spatial region. This produces a screened space-charge field due to the charge compensation. As a consequence, the index grating change is negligible, even at high $m$ values, and the photorefractive effect could not be produced in this kind of material. Therefore, the presence of material with the same electron and hole conductivity order could be bad if higher index changes are required. One can see from the plots that the gratings are screened since their strengths are relatively small. For the fundamental grating, the value of the Debye screening length, $\Lambda_{D}$, remains around the single-level model value [9] but, for higher harmonics, a peculiar behavior is observed: $\Lambda_{D}$ is shifted toward lower spacing values, as well as the harmonic gratings tend to appear more strongly. Finally, the harmonic grating behavior is 


\section{Harmonic Amplitudes E, (KV/cm)}
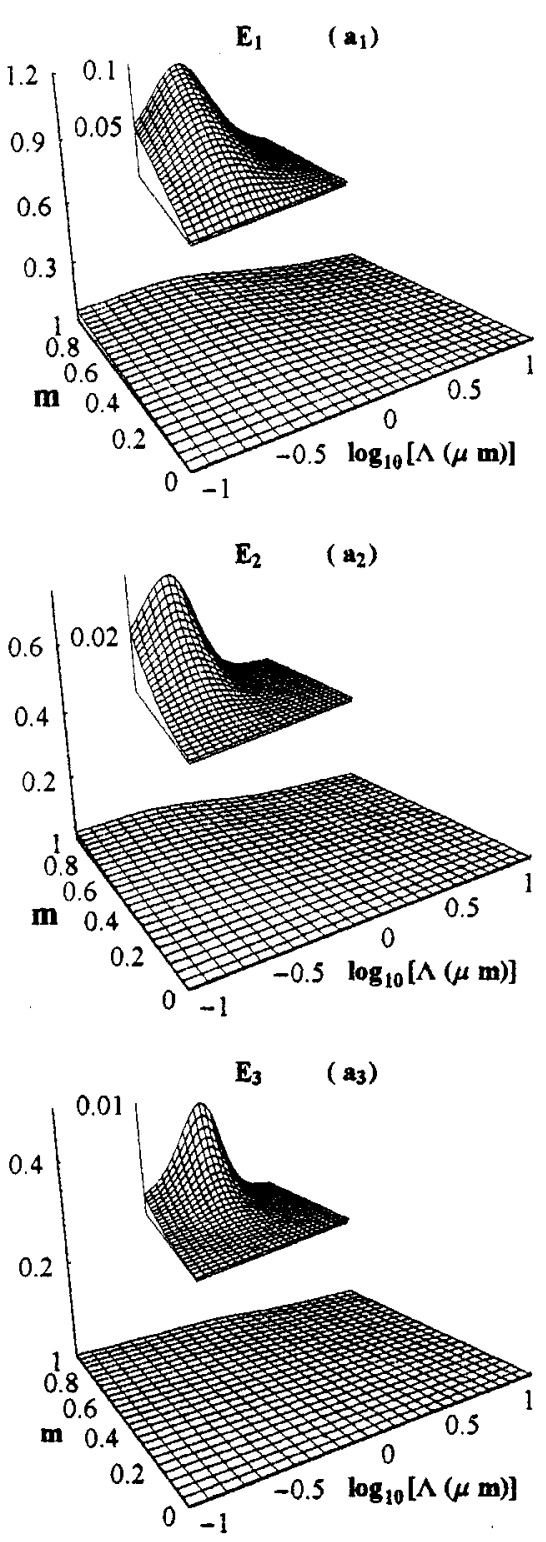
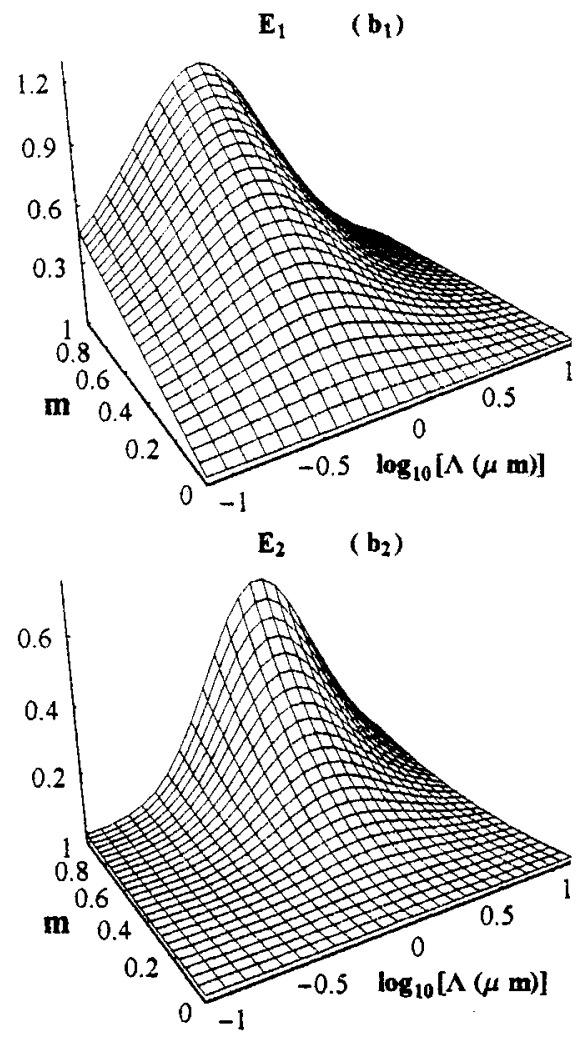

$\mathbf{E}_{3} \quad\left(\mathbf{b}_{3}\right)$

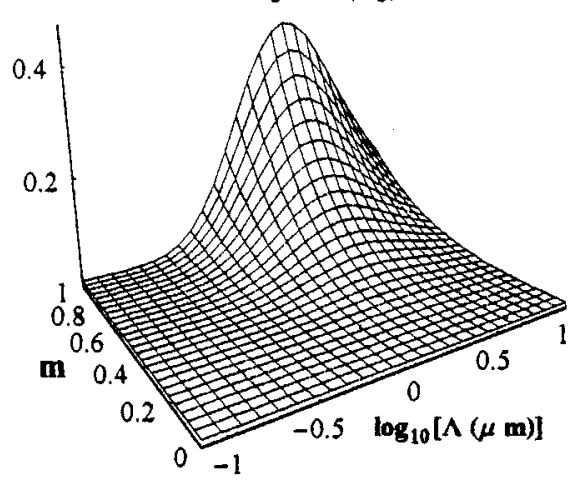

Fig. 1. Surfaces representing the fundamental, second, and third harmonic amplitude gratings versus the modulation depth and grating spacing within the range commonly employed for different relative concentrations of donor and acceptor densities. $\left(\mathrm{a}_{i}\right) N_{A}^{-}{ }_{\mathrm{cq}}=10^{16} \mathrm{~cm}^{-3}$ and $N_{A}=10^{17} \mathrm{~cm}^{-3}$, for a fixed value of $N_{D c q}^{+}=10^{16} \mathrm{~cm}^{-3}$ and $\left(\mathrm{b}_{i}\right) N_{A c q}^{-}=8 \times 10^{14} \mathrm{~cm}^{-3}$ and $N_{A}=5 \times 10^{18} \mathrm{~cm}^{-3}$, for a a fixed value of $10^{16} \mathrm{~cm}^{-3}$ for $N_{D \mathrm{cq}}^{+}$. There is not applied field.

analyzed for relatively small values of thermal ionized acceptors $N_{A \text { eq }}^{-} \ll N_{D \text { eq }}^{+}\left[\right.$Fig. $\left.1\left(\mathrm{~b}_{i}\right)\right]$. Due to the small amount of ionized acceptor traps, the redistribution of holes in acceptor traps is inefficient to induce the photorefractive effect. The gratings are mainly formed by electron redistribution in donor traps, since the hole-grating does not influence the photorefractive dynamics, and the harmonic amplitude surfaces are similar to the single-level model [9], as is expected. It is verified that for relatively low values of $N_{A \text { eq }}^{-}$, the harmonic amplitudes do not change for different values of total acceptor density $N_{A}$. Fig. 1 also shows how the harmonic amplitudes depend on the modulation depth $m$. The analysis in terms of $m$ is very important since the higher photorefractive harmonic gratings and certain nonlinear phenomena were observed for high values of this parameter [1], [4]. The linear, quadratic and cubic dependence of the fundamental, second and third harmonic amplitude versus $m$ for arbitrary modulation depth, grating spacing, and ionized and nonionized impurity density parameters, is clearly observed in Fig. 1. These peculiar results are similar to those obtained for the single-level [9] and shallow trap model [18]. In general, we prove that $E_{\nu} \propto m^{\nu}$, by using the induction complete principle. To emphasize the relative importance of the harmonic gratings $E_{\nu}$ in the usual experimental $(\Lambda, m)$ region, we analyze the ratio $E_{\nu} / E_{1}(\nu=2,3)$, which is plotted in Fig. 2. Some classical features, which appeared in the one-level and shallow trap model, are apparent in this model. It is shown that for the linear region, 


\section{$\mathbf{E}_{2} / \mathbf{E}_{1}$}

$\left(a_{1}\right)$

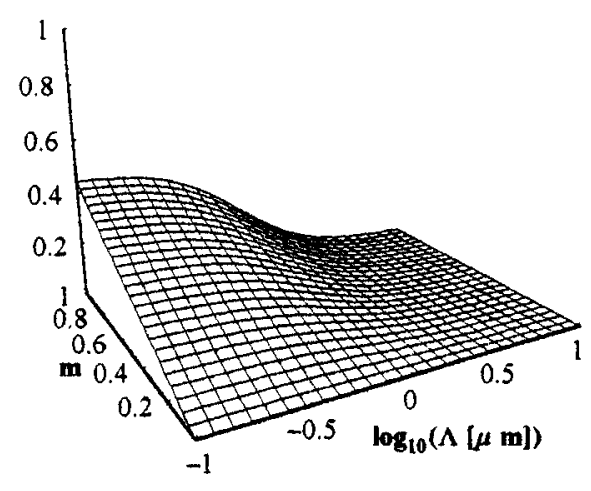

$\left(b_{1}\right)$

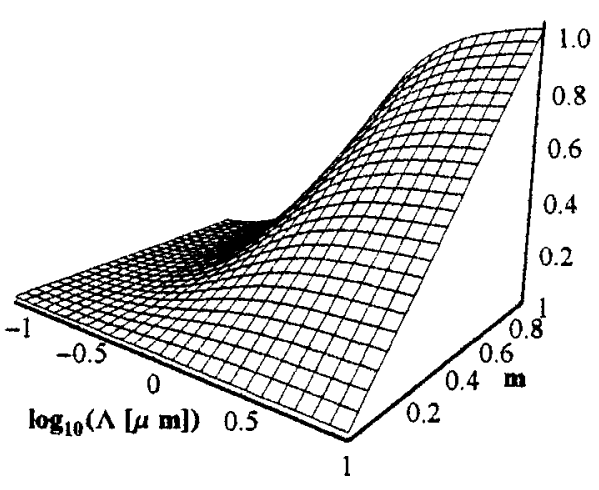

\section{$\mathbf{E}_{3} / \mathbf{E}_{1}$}
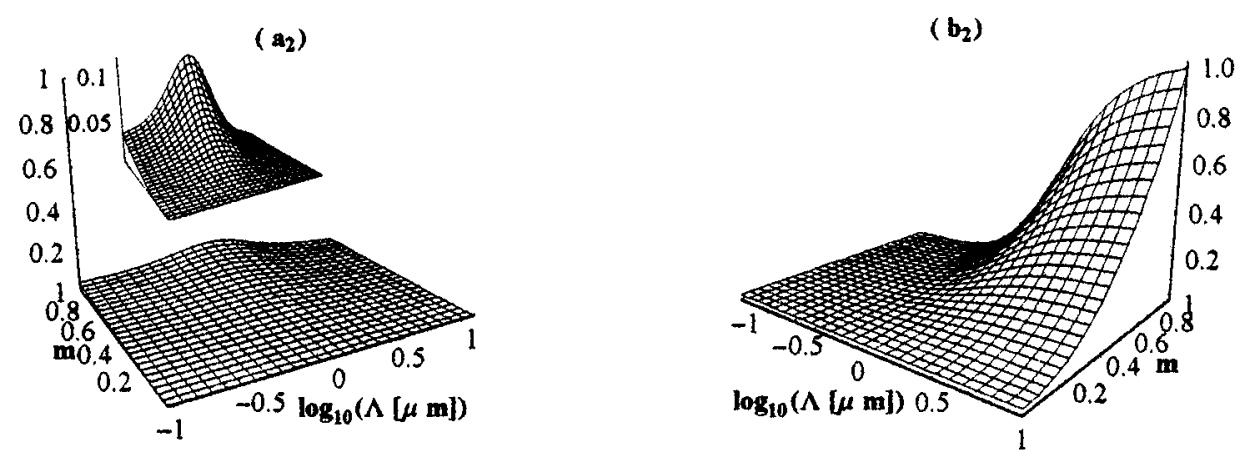

Fig. 2. Surfaces representing the second and third harmonic amplitudes normalized to the fundamental amplitude versus the modulation depth and grating spacing. Parameter values are given in Fig. 1.

$m \ll 1$, regardless of the spacing value, the highest harmonic gratings are negligible in comparison with the fundamental grating independently of $N_{A \text { eq }}^{-}$and $N_{A}$ values. Nonlinearities do not appear in this region. Three well-defined regions for the higher harmonic gratings relative to the fundamental grating can also be distinguished and identified as the linear, transition, and nonlinear regions. Within the electron-hole transport framework, the extent of each region in terms of $\Lambda$ depends on the ionized $N_{A \text { eq }}^{-}$and nonionized $N_{A}$ acceptors relative to donors strongly. A high concentration of acceptor traps produces a strong redistribution of holes. Consequently, the efficient formation of the internal field is a consequence of this charge carrier since the redistribution of electrons into donor traps remains screened. The harmonic components become important in an enlarged region of $\Lambda$, and the nonlinear region was extended toward lower spacings noticeably. In this nonlinear region, the importance of the higher order harmonics is strong even for small modulation values and, in consequence, depends strongly on both external parameters $\Lambda$ and $m$. Note that for $m$ values closer to one, the three higher harmonic amplitudes reach the fundamental grating. On the other hand, when the space-charge field is screened due to compensation of complementary electron-hole gratings [Fig. $2\left(\mathrm{a}_{i}\right)$ ], the higher harmonics are negligible against the fundamental grating in all $(\Lambda, m)$ regions. Only the second harmonic amplitude reaches a relatively noticeable value $(\succeq 0.4)$ for high $m$ and, curiously, for lower $\Lambda$ values. This happens because the $E_{2}$ Debye screening length $\Lambda_{D}$ is shifted toward lower spacing with respect to the screening length of fundamental amplitude as observed from Fig. 1. Afterwards, the Debye length screening of each higher order grating is shifted toward the highest spacing with respect to the fundamental order, and the maximum values of the $E_{\nu} / E_{1}$-surface are also shifted toward larger spacing. Finally, it is verified that for relatively low acceptor density $N_{A \text { eq }}^{-} \ll N_{D \text { eq }}^{+}\left[\right.$Fig. $\left.2\left(\mathrm{a}_{i}\right)\right]$, the ratios $E_{\nu} / E_{1}$ coincide with the classical result for the single-level model [9], since the redistribution of holes into these traps are negligible compared with the redistribution of electrons into donor traps, and nonlinear effects due to these centers do not appear. These results do not depend on the total acceptor density $N_{A}$ relative to donor density $N_{D}$. In short, the model predicts that the linear and nonlinear regimes depend not only on the modulation and the spacing, as in the single-level model, but also on the acceptor species. This suggests that an analysis of nonlinear response would be incomplete without including a detailed analysis of the complete set of these parameters.

The harmonic gratings behavior was studied on the external field to complete the analysis. Fig. 3 shows the dependence of the fundamental, second, third, and fourth harmonic amplitudes for $m=1$. Each row corresponds to different $\Lambda$-regions: $\Lambda=0.1 \mu \mathrm{m}$; in the figures $i=1, \Lambda=0.1 \mu \mathrm{m} ; i=2, \Lambda=$ $1 \mu \mathrm{m} ; i=3, \Lambda=10 \mu \mathrm{m}$. Each column corresponds to different concentrations of acceptor species, and their numerical 
$\Lambda=0.4 \mathrm{~m} \quad\left(a_{1}\right)$
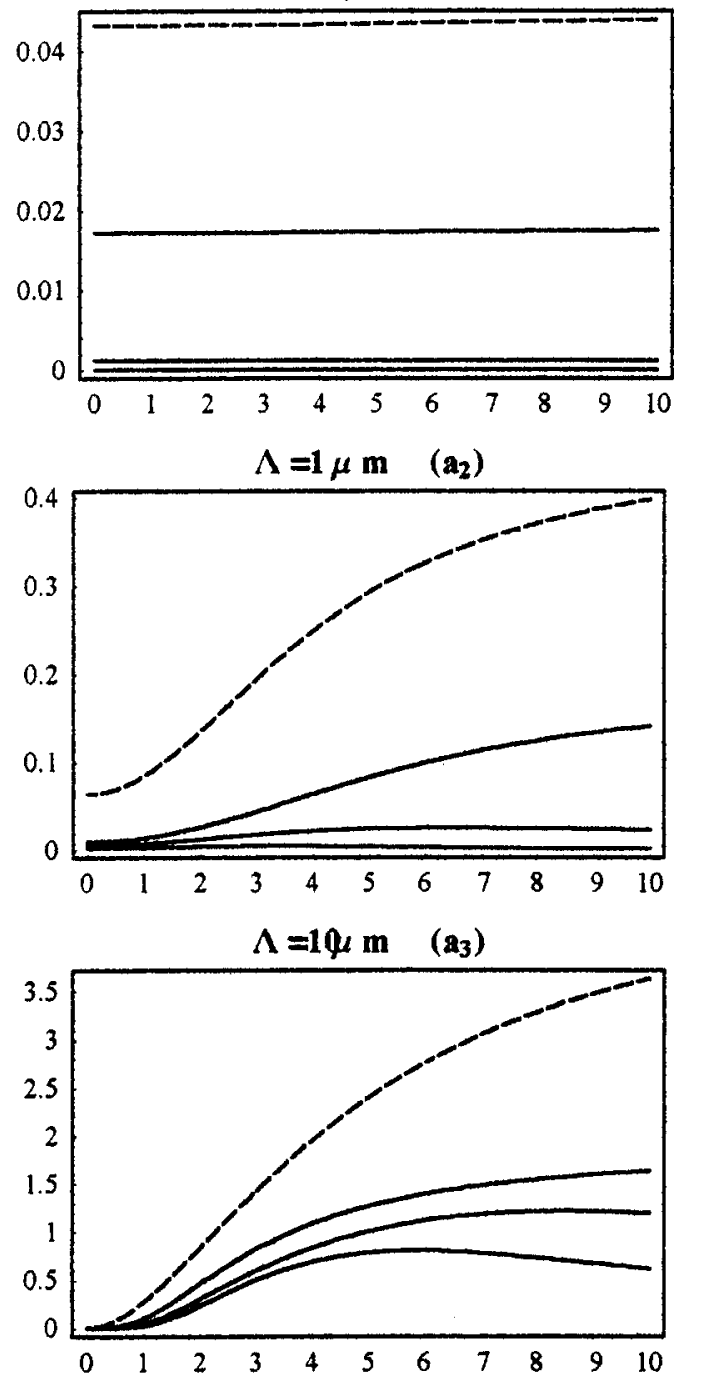

$\left(b_{1}\right)$
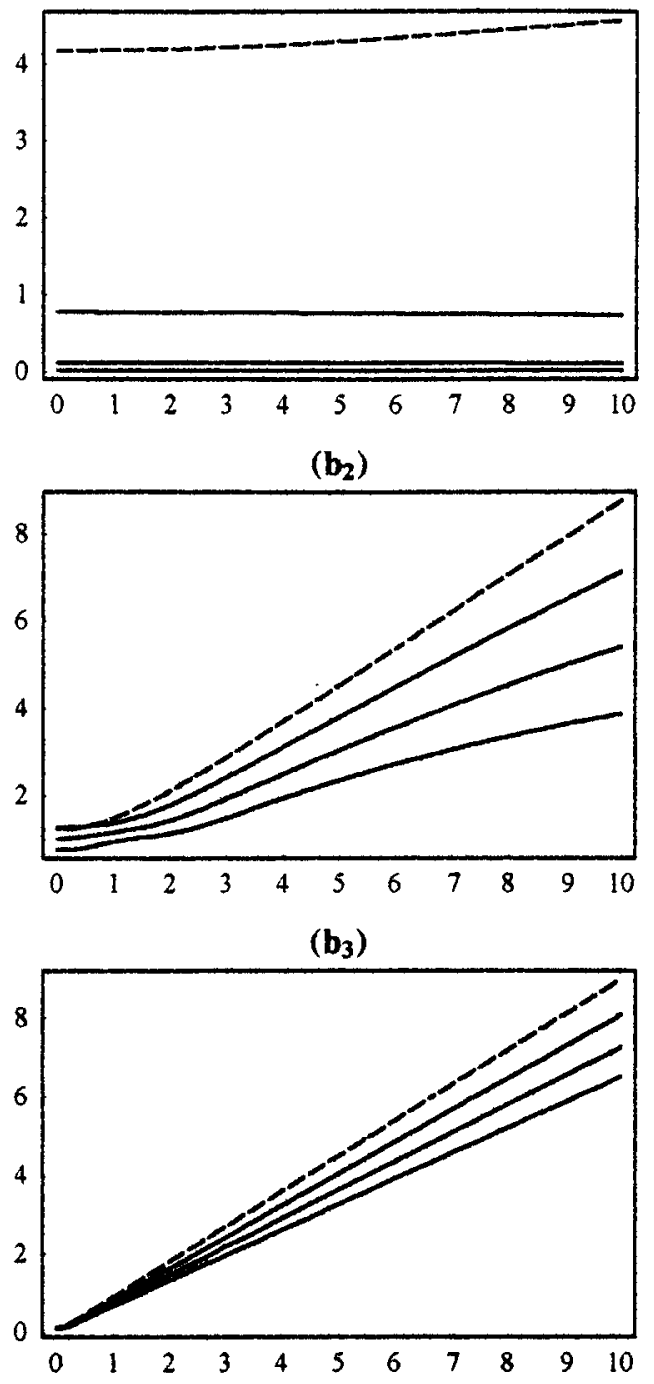

Fig. 3. Amplitudes of the first four higher order harmonic gratings in terms of the applied field. Dashed curve line: fundamental harmonic amplitude. Solid lines: second, third, and fourth harmonics. The rows represent the three different regions in $\Lambda$. The acceptor and donor densities are similar to those of Fig. 1 for $\left(\mathrm{a}_{i}\right)$ and $\left(b_{i}\right)$. The modulation depth value is $m=1$ in all cases.

values are given in Fig. 3. For $N_{A \text { eq }}^{-} \ll N_{D \text { eq }}^{+}$, the same behavior is verified as in the Kukhtarev model. Nevertheless, for $N_{A \text { eq }}^{-} \approx N_{D \text { eq }}^{+}$, the field amplitudes are screened as is expected. In terms of $\Lambda$, for $\Lambda \ll \Lambda_{D}$ [in Fig. 3( $\left.\mathrm{a}_{1}\right)$ and $\left(\mathrm{b}_{1}\right)$ ], the applied field does not change the amplitudes of the fundamental and harmonic gratings, for arbitrary values of $N_{A}$ and $N_{A \text { eq }}^{-}$. It means that the harmonics are negligible with regard to the fundamental amplitude. The region $\Lambda \approx \Lambda_{D}$ is shown in Fig. 3( $\left.\mathrm{a}_{2}\right)$ and $\left(\mathrm{b}_{2}\right)$. Note that the fundamental and harmonic amplitudes grow sublinearly, with the fundamental slope steeper than the harmonic ones when the applied field increases. Note that the behavior of the fundamental grating becomes more linear for higher values of $E_{0}$ and higher $N_{A \text { eq }}^{-}$as compared with $N_{D \text { eq }}^{+}$. In the region $\Lambda \gg \Lambda_{D}$, the increase of the harmonic gratings becomes outstanding as $E_{0}$ increases, as can be seen from Fig. 3( $\left.\mathrm{a}_{3}\right)$ and $\left(b_{3}\right)$. For high values of the applied field, the sublinear behavior of the harmonics with regard to the fundamental grating for low $N_{A \text { eq }}^{-}$is apparent. But, for higher concentrations of $N_{A \text { eq }}^{-}$, all the harmonics exhibit a linear behavior, as Fig. 3( $\left.b_{3}\right)$ shows. A strong screening of the photorefractive field appears when the acceptor density is similar to the donor density, as Fig. 3( $\left.a_{3}\right)$ indicates. The nonlinear response of the photorefractive medium is stronger for smaller values of the applied field, a result similar to the Kukhtarev [9] and shallow trap models [18].

\section{B. Space-Charge Field Profile}

The space-charge field is formed by the contribution of the harmonic components as it was early predicted by the pioneer work of Alphonse et al.[2]. They established that the sinusoidal profile loss of the space-charge field is a consequence of the contribution of the higher harmonics to the total internal field. Consequently, the nonsinusoidal modulation of the refractive index is due to the nonlinear effect. As a consequence, numerous papers have attempted to emphasize the nonlinear response by analyzing the spatial distribution along a grating period within the one-level model framework. Nevertheless, despite the experimental evidence that indicates the existence of many materials 

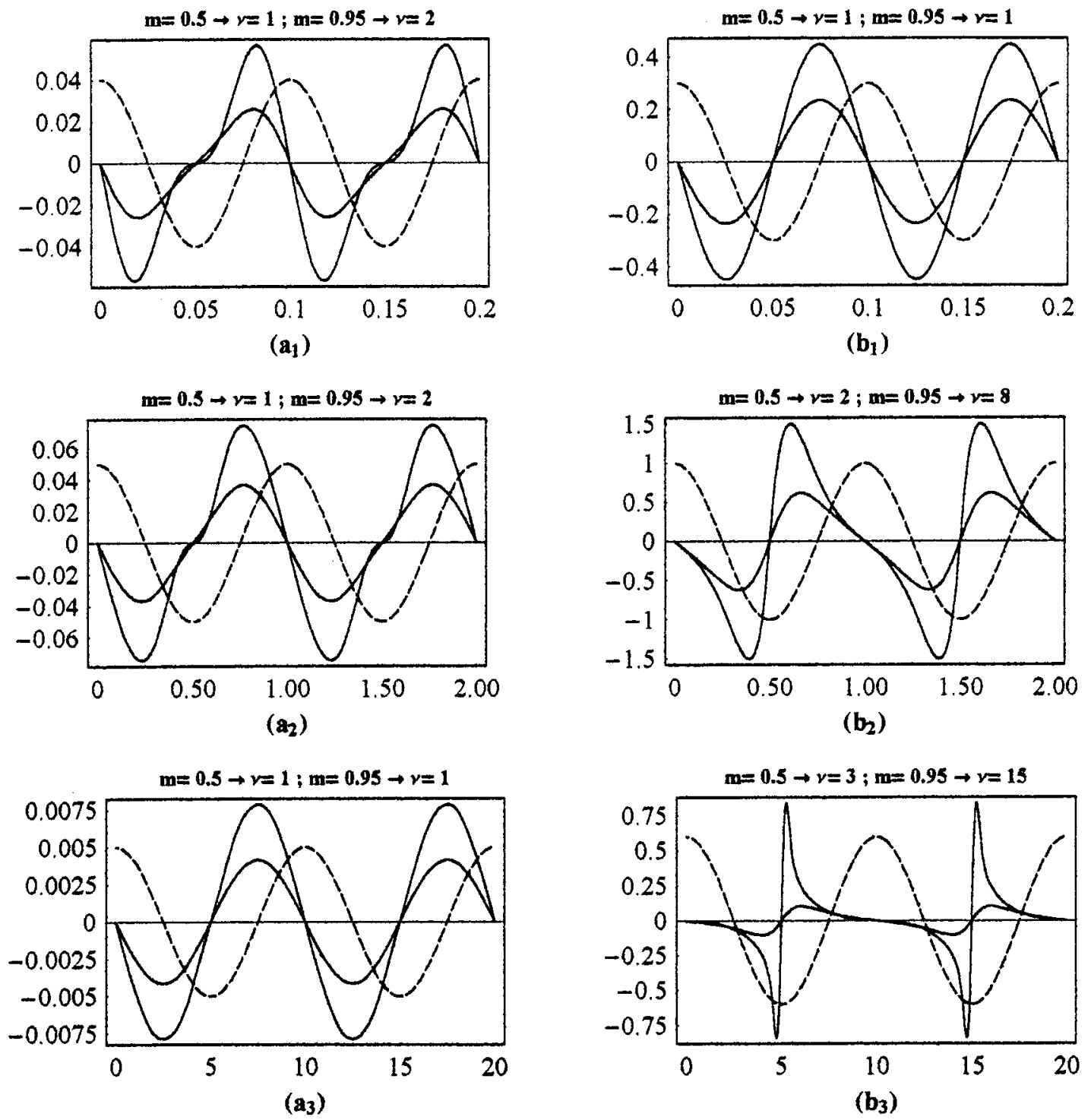

Fig. 4. Space-charge field profile along two grating periods without applied field. Dashed lines: excitation pattern profile. Solid curves: profile for $m=0.95$ and $m=0.5$, respectively. The number $\nu$ indicates the size of Fourier expansion components needed for the space-charge field convergence for each $m$ value. The rows represent the three different region in $\Lambda$. The acceptor and donor density values are similar to those of Fig. 1 for $\left(a_{i}\right)$ and $\left(b_{i}\right)$.

with an electron-hole transport mechanism, there are few papers studing the grating behavior within this framework, but they employ only approximate methods. A rigorous analysis of the mentioned profile is possible only with the exact knowledge of the $\nu$-order amplitudes and phases of the harmonic gratings from an analytic expressions. In this section, we describe the space charge field profile by using the electron-hole transport model given by (21)-(23), which enables us to predict the $E_{\mathrm{SC}}$-profile from the analysis of the convergence of the Fourier expansion (11). In Fig. 4 , the $E_{\mathrm{SC}}$-profile is plotted along two grating periods for several values of the modulation depth in the three $\Lambda$-intervals studied: linear $(\Lambda=0.1 \mu \mathrm{m}$, subindex $i=1)$, transition $(\Lambda=1 \mu \mathrm{m}, i=2)$ and nonlinear $(\Lambda=10 \mu \mathrm{m}, i=3)$, without an applied field. Each column corresponds to different values of the ionized acceptors and the total density with regard to the donors. Fig. $4\left(\mathrm{a}_{i}\right)$ shows the spatial profile for $N_{A \text { eq }}^{-} \approx N_{D \text { eq }}^{+}$ and $\left(\mathrm{b}_{i}\right)$ for $N_{A \text { eq }}^{-} \gg N_{D \text { eq }}^{+}$, observing their different behaviors. For $N_{A \text { eq }}^{-} \approx N_{D \text { eq }}^{-}$, a peculiar behavior is evident for the elec- tron-hole model established in Figs. 1 and 2: the space-charge field loses its sinusoidal profile in the smaller spacing region [Fig. 4(a $\left.a_{1}\right)$, being noticeable in the relative influence of the second harmonic. Its relative amplitude decreases when $\Lambda$ increases [Fig. 4( $\left.\mathrm{a}_{2}\right)$ ], and it becomes negligible in the high $\Lambda$-region. As a consequence, the sinusoidal profile is observed [Fig. 4(a $\left.\mathrm{a}_{3}\right)$ ]. However, in all the cases, the grating amplitudes are relatively low due to the competition among the $e-h$ gratings. On the other hand, and in accordance with preceding section, when the redistribution of one of the carriers is dominant, the results coincide with the Kukhtarev model [9]: the region of smaller spacings [Fig. $4\left(b_{1}\right)$ ] corresponds to the linear region where the profile is sinusoidal. Only the component $\nu=1$ is necessary, even for relatively high values of $m$. In the $\Lambda$-region [Fig. $4\left(\mathrm{~b}_{2}\right)$ ], the space-charge field slightly loses its sinusoidal profile which indicates the growth of middle harmonic gratings. A $\pi / 2$-shift between the grating and the interference fringes (dashed lines) is also observed. Finally, the region of higher spacings [Fig. 4(b $\left.b_{3}\right)$ ] 

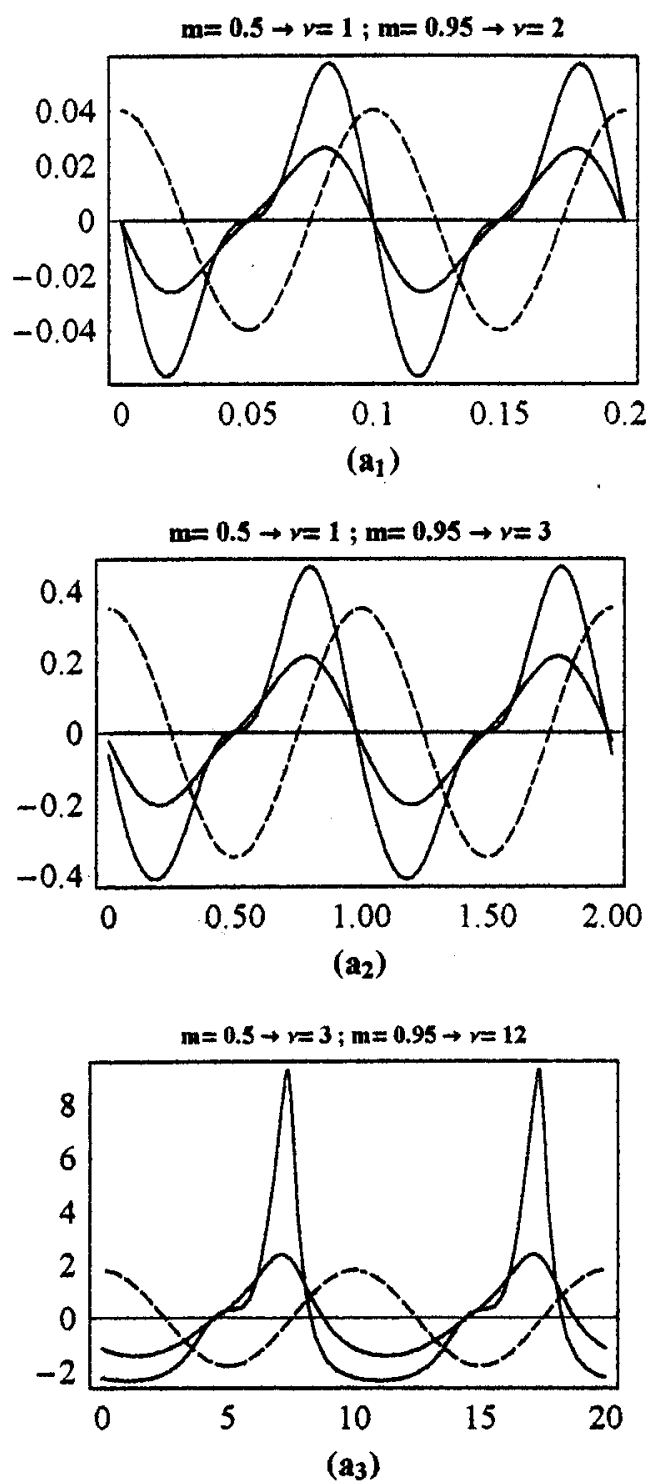
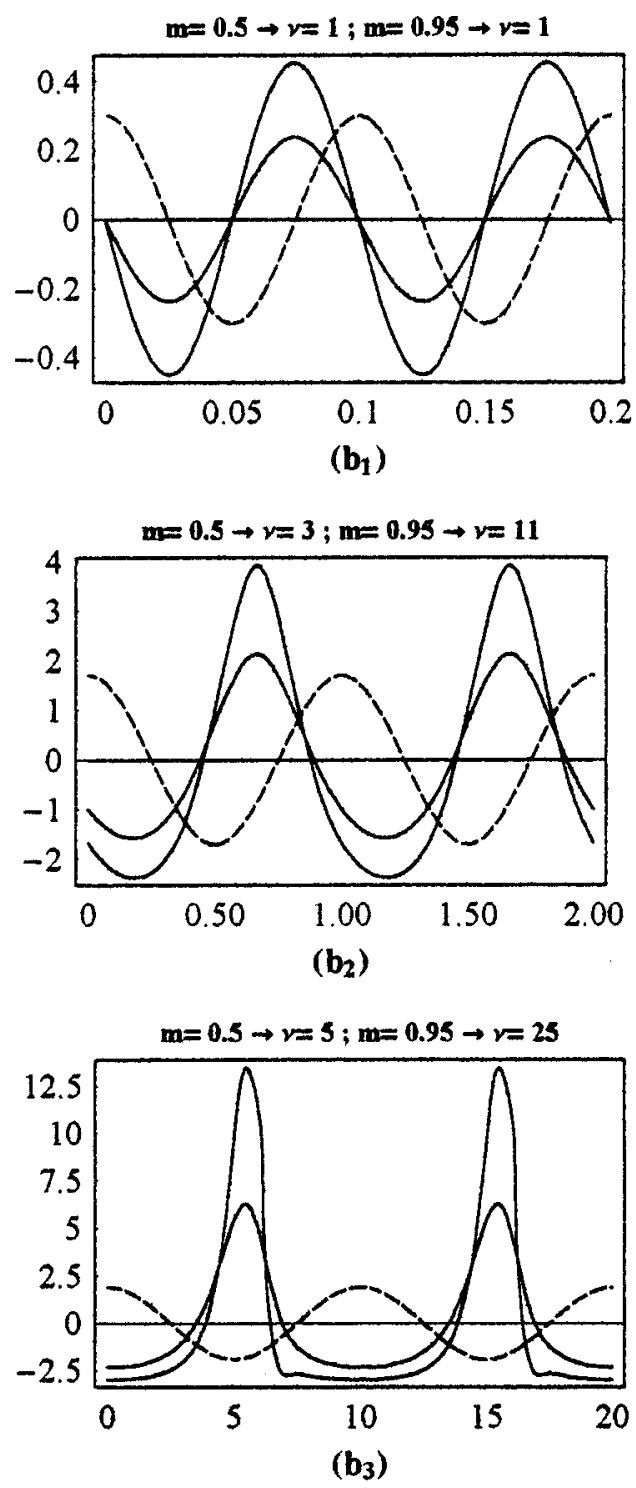

Fig. 5. Space-charge field profile with the same parameters as those employed in Fig. 4 but with an applied field of $5 \mathrm{kV} / \mathrm{cm}$.

is identified with the nonlinear region. In this case, the number of harmonic components needed to synthesize the space-charge field is high and the sinusoidal profile is completely lost.

The spatial distribution of the space charge field versus the applied field is shown in Fig. 5. Each row corresponds to the above mentioned $\Lambda$-regions, and each column corresponds to acceptor and donor density values. An antisymmetric behavior around the point at which the field is null is broken as Fig. 5 shows. This point changes with the modulation $m$ and the positive values of $E_{\mathrm{SC}}$ are now different in comparison with the negative values. This asymmetry depends on the external field direction, which defines a privileged transport direction. Therefore, if one needs to store a large number of gratings, then it is necessary not only to move to high modulation and high spacings, but also to avoid materials with similar electron and hole photoconductivities.

\section{CONCLUSION}

The behavior of the steady-state of photorefractive harmonic gratings was derived without any simplifications by solving in exact form the material equations within an electron-hole transport framework. The analysis was done by employing material parameters values corresponding to BSO crystals and primary external parameters, such as the average excitation intensity, modulation depth, grating spacing, and the applied electric field within the experimental ranges commonly used. It was found that the higher harmonic amplitudes do not depend on the average intensity of the fringe pattern in a typical $\mathrm{CW}$ range. In terms of the grating spacing, and in agreement with the single-level model, the three regions of quite different behavior are identified and analyzed. Within the electron-hole transport framework, the extent of each region in terms of $\Lambda$ strongly depends on the ionized $N_{A \text { eq }}^{-}$and nonionized $N_{A}$ acceptor species relative to the donor species. If the acceptor density is much greater or smaller than the donor density, the linear region spreads out toward the lowest spacing, the nonlinear region extends toward the highest spacing, and the middle region is located in-between, as in the Kukhtarev model. However, for similar concentrations, the nonlinear region is shifted toward smaller spacing with respect to the linear region, 
since the Debye screening length of the higher component is shifted toward smaller spacings. Besides, the $E_{\nu}$ harmonic gratings dependence in terms of the modulation depth is rigorously established as $m^{\nu}$ as in the single level model. In terms of the applied field, the nonlinear response is apparent for small values of this parameter. On the other hand, the electron-hole competition, associated with similar donor and acceptor densities, can be deleterious for the grating recording, due to charge compensation produced by the additional charge carrier that screens the internal space-charge field.

\section{REFERENCES}

[1] J. V. Alvarez-Bravo, M. Carrascosa, and L. Arizmendi, "Experimental effects of light intensity modulation om the reading and erasure of holographic gratings in BSO crystals," Opt. Commun., vol. 103, pp. 22-28, 1993.

[2] G. A. Alphonse, R. C. Alig, D. L. Staebler, and W. Phillips, "Time dependent characteristics of photoinduced space-charge field and phase holograms in lithium niobate and other photorefractive media," $R C A$ Rev., vol. 36, pp. 213-229, 1975.

[3] D. A. Temple and C. Warde, "High-order anisotropic diffraction in photorefractive crystals," J. Opt. Soc. Amer. B, vol. 5, pp. 1800-1805, 1988

[4] Y. H. Lee and R. W. Hellwarth, "Spatial harmonics of photorefractive in barium titanate crystals," J. Appl. Phys., vol. 71, pp. 916-923, 1992.

[5] J. E. Millerd, E. M. Garmire, M. B. Klein, B. A. Wechsler, F. P. Strohkendl, and G. A. Brost, "Photorefractive response at high modulation depths in $\mathrm{Bi}_{12} \mathrm{SiO}_{20}$," J. Opt. Soc. Amer. B, vol. 9, pp. 1449-1453, 1992

[6] J. P. Huignard and B. Ledu, "Collinear Bragg diffraction in photorefractive $\mathrm{Bi}_{12} \mathrm{SiO}_{20}$," Opt. Lett., vol. 7, pp. 310-312, 1982.

[7] F. Vachss and L. Hesselink, "Selective enhancement of spatial harmonics of photorefractive gratings," J. Opt. Soc. Amer. B, vol. 5, pp. 1814-1821, 1988.

[8] E. Serrano, V. Lopez, M. Carrascosa, and F. Agullo-Lopez, "Steady-state photorefractive gratings in $\mathrm{LiNbO}_{3}$ for strong light modulation depths," IEEE J. Quantum Electron., vol. Q-30, pp. 875-880, 1994.

[9] P. Vaveliuk, B. Ruiz, and N. Bolognini, "Analysis of the steady-state photorefractive harmonic gratings," Phys. Rev. B, vol. 59, pp. 10985-10 991, 1999.

[10] D. Mahgerefteh and J. Feinberg, "Explanation of the apparent sublinear conductivity in photorefractive barium titanate," Phys. Rev. Lett., vol. 64, pp. 2195-2198, 1990.

[11] I. Biaggio, M. Zgonik, and P. Gunter, "Build-up and dark decay of transient photorefractive gratings in reduced $\mathrm{KNbO}_{3}$," Opt. Commun., vol. 77, pp. 312-316, 1990.

[12] G. Pauliat and G. Roosen, "Photorefractive effect generated in sillenite crystals by picosecond pulses and comparison with the quasicontinuous regime," J. Opt. Soc. Amer. B, vol. 7, pp. 2259-2267, 1990

[13] P. Tayebati, "The effect of shallow trap on the dark storage of photorefractive grating in $\mathrm{Bi}_{12} \mathrm{SiO}_{20}$," J. Appl. Phys., vol. 70, pp. 4082-4094, 1991.

[14] P. Nouchi, J. P. Partanen, and R. W. Hellwarth, "Simple transient solutions for photoconduction and the space-charge field in a photorefractive material with shallow traps," Phys. Rev. B, vol. 47, pp. 15 581-15 587, 1993.

[15] J. Li, X. H. Li, F. Y. Lu, H. F. Wang, S. Z. Yuan, Y. Zhu, and X. Wu, "Erasure of photorefractive gratings in barium titanate," J. Appl. Phys., vol. 76, pp. 7541-7544, 1994

[16] G. Le Saux and A. Brun, "Photorefractive material response to short pulse ilumination," IEEE J. Quantum Electron., vol. Q-23, pp. $1680-1688,1987$

[17] P. Tayebati and D. Mahgerefteh, "Theory of the photorefractive effect for $\mathrm{Bi}_{12} \mathrm{SiO}_{20}$ and $\mathrm{BaTiO}_{3}$ with shallow traps," J. Opt. Soc. Amer. B, vol. 8, pp. 1053-1064, 1991.

[18] P. Vaveliuk, B. Ruiz, N. Bolognini, G. A. Torchia, and J. Fernandez, "Steady-state photorefractive harmonic gratings within the shallow-trap model," J. Optics A, submitted for publication.

[19] N. Barry and M. J. Damzen, "Two beams coupling and response-time measurements in barium titanate using high intensity," J. Opt. Soc. Amer $B$, vol. 9, pp. 1488-1492, 1992

[20] M. J. Damzen and N. Barry, "Intensity-dependent hole-electron competition and photocarrier saturation in $\mathrm{BaTiO}_{3}$ when using intense laser pulses," J. Opt. Soc. Amer. B, vol. 10, pp. 600-606, 1993.

[21] C. Medrano and P. Gunter, Insulating Materials for Optoelectronics, F. Agullo-Lopez, Ed, Singapore: World Scientific, 1995, ch. 10.
[22] P. Vaveliuk, B. Ruiz, R. Duchowicz, and N. Bolognini, "Theoretical analysis of the photocurrent dark decay in photorefractive media," IEEE J. Quantum Electron., vol. 36, pp. 692-697, 2000.

[23] L. Boutsikaris and F. Davidson, "Perturbative analysis of higher-order photorefractive gratings in InP:Fe," Opt. Commun., vol. 105, pp. 411-420, 1994.

[24] L. Boutsikaris, S. Mailis, and N. A. Vainos, "Determination of the photorefractive parameter of $\mathrm{Bi}_{12} \mathrm{SiO}_{20}$ by study of the dynamic behavior of complementary gratings," J. Opt. Soc. Amer. B, vol. 15, pp. 1042-1051, 1998.

[25] A. Shumelyuk, S. Odoulov, and G. Brost, "Nearly degenerate two-beam coupling in photorefractive crystals with two species of movable carriers," J. Opt. Soc. Amer. B, vol. 15, pp. 2125-2131, 1998.

[26] _ " "Multiline coherent oscillation in photorefractive crystals with two species of movable carriers," Appl. Phys. B, vol. 68, pp. 959-966, 1999.

[27] G. C. Valley, "Simultaneous electron/hole transport in photorefractive materials," J. Appl. Phys., vol. 59, pp. 3363-3366, 1986.

[28] P. Vaveliuk, B. Ruiz, N. Bolognini, and J. Fernandez, "Transient behavior of the photorefractive space-charge field," Phys. Rev. B, vol. 62, pp. $4511-4518,2000$

[29] L. M. Bernardo, J. C. Lopes, and O. D. S. Soares, "Hole-electron competition with fast and slow gratings in $\mathrm{Bi}_{12} \mathrm{SiO}_{20}$ crystals," Appl. Opt. vol. 29 , pp. $12-14,1990$

[30] S. Zhivkova and M. Miteva, "Holographic recording in photorefractive crystals with simultaneous electron-hole transport and two active centers," J. Appl. Phys., vol. 68, pp. 3099-3102, 1990.

Pablo Vaveliuk received the Ph.D. degree in physics from the Universidad Nacional de La Plata, La Plata, Argentina, in 2000.

He joined the Centro de Investigaciones Opticas, La Plata, Argentina, in 1995. His research activities have included nonlinear optics, photoacoustic, photorefractive effect and crystals. He holds a fellowship from the National Research Council of Argentina (CONICET)

Beatriz Ruiz received the M.S. degree in mathematics from the Universidad Nacional de La Plata, La Plata, Argentina, in 1961

She is currently a Professor of Mathematics at Universidad Nacional de La Plata, and a Research Assistant in the Centro de Investigaciones Opticas, La Plata, Argentina.

Oscar Martinez Matos received the M.S. degree in physics from the Universidad Complutense de Madrid, Madrid, Spain, in 1996, and is currently working toward the Ph.D. degree at Universidad Nacional de La Plata, La Plata, Argentina.

He began research with the Centro de Investigaciones Opticas de La Plata, La Plata, Argentina, in 1999. His research includes laser spectroscopy, nonlinear optics, photoacoustics, and photorefractive crystals. He holds a fellowship from FOMEC (Argentina)

Gustavo A. Torchia received the Ph.D. degree in physics from the Universidad Nacional de La Plata, La Plata, Argentina in 2000.

He has been with the Centro de Investigaciones Opticas de La Plata, La Plata, Argentina, since 1996, where he teaches physics. He was previously a Researcher at the Universidad Autónoma de Madrid, Madrid, Spain. His research activities have included laser spectroscopy, photoacoustic, insulator materials, new laser devices, and photorefractive crystals. He holds a fellowship from the National Research Council of Argentina (CONICET).

Nestor Bolognini received the Ph.D. degree in physics from the Universidad Nacional de La Plata, La Plata, Argentina, in 1981.

He joined the Centro de Investigaciones Opticas, La Plata, Argentina, in 1977. He was later a Researcher at the University of Stuttgart, Stuttgart, Germany, and the Universidad Autonoma de Madrid, Madrid, Spain. His research activities have included speckle techniques, holography, and Fourier optics. He is currently a Researcher with the National Research Council of Argentina (CONICET), as well as a Professor of physics and Ph.D. thesis adviser at Universidad Nacional de La Plata. He is currently involved in image processing using photorefractive materials. 